\title{
The People's League of Health trial
}

\author{
Sjúrður Fróði Olsen
}

J R Soc Med 2006;99:44-45

From The James Lind Library [www.jameslindlibrary.org]

The ambitious objective of the People's League of Health, according to the pamphlet published soon after the League was instituted in 1917, was no less than 'to raise the standard of health of the British nation'. ${ }^{1}$ The League's founder and honorary organizer was Miss Olga Nethersole, a former actress.

During the late 1920s and early 1930s, Olga Nethersole approached a number of people within the medical establishment for support, but was rebuffed in no uncertain terms by Sir Walter Fletcher, Secretary of the Medical Research Council. In letters that can be inspected in the archive of the Medical Research Council, Fletcher warned colleagues who had been invited to join 'a fantastic "SubCommittee on Bovine Tuberculosis" appointed by the League', that it was becoming 'a great nuisance', with 'a mixed lot of charlatans and advertisers (with one or two honest men who have been had for mugs and ought to have known better)'; and adding that one of Miss Nethersole's allies (Tippet) was a 'tiresome busybody' and an 'illbalanced ignoramus reacting upon (her) benevolent but uninformed enthusiasm'. A letter written by Fletcher on 23 May 1930 to one of his staff — Dr Stanley Griffith — opens with the advice 'Do tell Olga Nethersole to go to blazes!'.

In spite of this opposition from some elements of the medical establishment, in 1935, an executive team for the People's League of Health pregnancy supplementation trial was established, with impetus from Dame Louise McIlroy DBE MD DSc. The team consisted of 15 people, including obstetricians, a pharmacist, a biochemist, and a statistician (W T Russell), and was chaired by the Professor of Obstetrics and Gynaecology at the British Postgraduate Medical School. The stated aim of the trial was to assess whether additions of vitamins and minerals to the diet of pregnant women would benefit the course of pregnancy and labour, and the health of the newborn child.

This team certainly accomplished a great deal. An initial pilot study of the dietary intake of 1000 women over 1 week was used to assess which constituents to include in the dietary supplement to be tested in the trial. The main trial

Maternal Nutrition Group, Danish Epidemiology Science Centre, Statens Serum Institut, Artillerivej 5, DK-2300 Copenhagen S, Denmark was undertaken during 1938-1939 at 10 London hospitals. Five thousand pregnant women participated in it, and, for more than 50 years, the study remained by far the largest properly controlled trial of a dietary intervention in pregnancy.

Allocation to treatment groups in the trial was achieved by alternate allocation, which, if strictly adhered to, abolishes allocation bias. ${ }^{2}$ Although it is impossible to be sure that there was strict adherence to the allocation schedule, the characteristics of the women in the comparison groups were similar, and there are no indications from the reports of the trial that alternation was not successful in abolishing allocation bias. [It is interesting that, during the planning phase of the League's trial, a much smaller but similar trial was conducted by Theobald, who invited pregnant women to draw blue or white beads from a box to decide which of them should be assigned to a dietary supplement and which to a control group. ${ }^{3}$ Although there is some uncertainty about the exact procedure that he used, this would seem a safer technique than alternation to ensure unbiased allocation to comparison groups. ${ }^{4,5}$ ]

The People's League of Health trial found that women who had received supplements during pregnancy were less likely to have developed 'toxaemia' and to have delivered early.

The study was reported in two simultaneous and nearly identical interim reports in 1942 in the BMJ and The Lancet, and in a final report published in 1946 in the Journal of Obstetrics and Gynaecology of the British Empire. ${ }^{6-8}$ An indication of the idealistic spirit of the team that designed and ran the trial is that the reports all named the League as the author (names and affiliations of the persons on the team were stated within the papers).

Despite the potential significance of these findings, reports of the trial received a mixed reception. The Chief Medical Officer of the time-Sir Wilson Jamesonaddressing a delegation from the trial committee, is reported to have said 'You have shown, I think for the first time, what can be done with supplements in place of foodstuffs; I do not think any other investigation comparable with this has been undertaken' (cited in letter from Olga Nethersole to Sir Edward Mellanby). The research community, by contrast, appears to have received the reports critically. 
A colleague and I have addressed these reactions (and other issues pertaining to the trial) in more detail elsewhere. ${ }^{9}$ Our conclusion is that the People's League of Health trial was an exceptional and well conducted study, which remains of great relevance today.

Acknowledgments I thank Iain Chambers for helpful discussions about the trial and the Medical Research Council for permission to access historical material. My research on maternal nutrition is supported by the March of Dimes Birth Defects Foundation.

\section{REFERENCES}

1 Nethersole O. The People's League of Health: The Inception of the League. London: Metchim \& Son, 1922
2 Chalmers I. Comparing like with like: some historical milestones in the evolution of methods to create unbiased comparison groups in therapeutic experiments. Int J Epidemiol 2001;30:1170-8

3 Theobald GW. The effect of calcium and vitamins A and D on incidence of pregnancy toxæmia. Lancet 1937;232:1397-9

4 Olsen SF. Electronic responses. The first clinical trial with true randomisation of individuals? eBMJ 1998;317:1220-3 [www.bmj.com/ cgi/eletters/317/7167/1220]

5 Olsen SF. Use of randomisation in early clinical trials. Theobald's trial in 1936 incorporated some aspects of randomisation. BMJ 1999; 318:1352

6 People's League of Health. Nutrition of expectant and nursing mothers. Lancet 1942;2:10-12

7 People's League of Health. The nutrition of expectant and nursing mothers in relation to maternal and infant mortality and morbidity. J Obstet Gynaecol Br Emp 1946;53:498-509

8 People's League of Health. Nutrition of expectant and nursing mothers. BMJ 1942;2:77-8

9 Olsen SF, Secher NJ. A possible preventive effect of low-dose fish oil on early delivery and pre-eclampsia: indications from a 50-year-old controlled trial. Br J Nutr 1990;64:599-609

\section{CORRECTION}

Tröhler U. Lind and scurvy: 1747 to 1795 . J R Soc Med 2005;98:519-22

In the second line of the final paragraph in column 1 on p. 521 of the November issue of JRSM, we inadvertently printed 'Thomas Cook' instead of James Cook. Thomas Cook was not born until 1808 — over 30 years after James Cook's second voyage! 